\title{
Phase-Based Road Detection in Multi-Source Images
}

S. K. Sengupta, A. S. Lopez, J. M. Brase, D. W. Paglieroni

June 17, 2004

International Geoscience and Remote Sensing Symposium (IGARSS)

Anchorage, AK, United States

September 20, 2004 through September 24, 2004 
This document was prepared as an account of work sponsored by an agency of the United States Government. Neither the United States Government nor the University of California nor any of their employees, makes any warranty, express or implied, or assumes any legal liability or responsibility for the accuracy, completeness, or usefulness of any information, apparatus, product, or process disclosed, or represents that its use would not infringe privately owned rights. Reference herein to any specific commercial product, process, or service by trade name, trademark, manufacturer, or otherwise, does not necessarily constitute or imply its endorsement, recommendation, or favoring by the United States Government or the University of California. The views and opinions of authors expressed herein do not necessarily state or reflect those of the United States Government or the University of California, and shall not be used for advertising or product endorsement purposes. 


\title{
Phase-Based Road Detection in Multi-Source Images ${ }^{1}$
}

\author{
Sailes K. Sengupta, Aseneth S. Lopez, James M. Brase and David W. Paglieroni \\ Lawrence Livermore National Laboratory \\ P.O. Box 808, L-290, Livermore, CA 94550 \\ email: sengupta2@Ilnl.gov vmail: 925-423-2369
}

\begin{abstract}
The problem of robust automatic road detection in remotely sensed images is complicated by the fact that the sensor, spatial resolution, acquisition conditions, road width, road orientation and road material composition can all vary. A novel technique for detecting road pixels in multi-source remotely sensed images based on the phase (i.e., orientation or directional) information in edge pixels is described. A very dense map of edges extracted from the image is separated into channels, each containing edge pixels whose phases lie within a different range of orientations. The edge map associated with each channel is de-cluttered. A map of road pixels is formed by re-combining the de-cluttered channels into a composite edge image which is itself then separately de-cluttered. Road detection results are provided for DigitalGlobe and TerraServerUSA images. Road representations suitable for various applications are then discussed.
\end{abstract}

\section{INTRODUCTION}

Roads (or more broadly, lines-of-communication) are entities commonly stored in Geographical Information Systems (GIS's). Unfortunately, because current images are not always available and manual road detection is labor-intensive, line-of-communication networks stored in GIS's are often incomplete or outdated. This situation can jeopardize the use of road data to guide the search for moving vehicles or to provide corroborative evidence in suspected detections of moving vehicles. It is thus important to be able to automatically detect roads in recently acquired remotely sensed images.

Pixel classification techniques are useful for detecting roads in multi-band images when the spectral signatures for most types of road pixels are known and the spectra for non-road pixels are distinguishable from the spectra for road pixels ([1]). While relatively simple and computationally efficient, these road detection methods lack robustness. They typically do not work well on monochrome images and are sensitive to changes in imaging sensor, time of day, season of year, weather conditions, etc.

Texture classification techniques seek image tiles whose textures resemble road textures. Since the orientation of road texture varies with road direction, it is important to use texture analysis techniques, such as those based on Gabor wavelets, that can take orientation into account ([2]-[4]). One might expect texture classifiers to be more robust than pixel classifiers, especially if the images are first pre-processed with a gradient or high-pass filter. However, they require example textures, which can vary significantly depending on the type of image, and type or width of the road.

Hough transform techniques, when applied to small rectangular image tiles, can be used to detect roads in edge images by seeking parallel lines separated by a prescribed amount ([5]). They are not wellsuited to detecting roads of arbitrary width. Also, because they are very sensitive to clutter, success requires the de-cluttered input edge images to be of very high quality.

An interesting alternative technique for detecting road pixels in multi-source remotely sensed images based on the phase (i.e., orientation or directional) information in edge pixels is described in Section II. The technique is designed to be robust across images acquired by various sensors under a variety of conditions. A very dense map of edges extracted from the image is separated into channels, each containing edge pixels whose phases lie within a different range of orientations. The edge map associated with each channel is de-cluttered. A map of road pixels is formed by re-combining the de-cluttered

1 This work was performed under the auspices of the U.S. Department of Energy by the University of California, Lawrence Livermore National Laboratory, under Contract No. W-7405-Eng-48. 
channels into a composite edge image which is itself then separately de-cluttered. Road detection results are provided for DigitalGlobe and TerraServerUSA images in Section III. Road representations suitable for various applications are then discussed in Section IV.

\section{PHASE-BASED ROAD DETECTION}

The road detector presented in this paper uses a dense edge image as input. Edges are traditionally detected either by seeking zero-crossings in Laplacian-of-Gaussian filtered images at locations of sufficient derivative amplitude ([6]) or by seeking local maxima of sufficient amplitude in the mathematical gradient of pixel gray-scale intensity ([7]). A simpler method (attributed to Prewitt) that pre-dates these techniques computes the "composite" directional derivative at each pixel as a weighted sum of vectors from the center of the pixel to the center of each of its 8 neighbors ([8]). For pixel $(x, y)$, the Prewitt directional derivative can be generalized to multi-band images as

$$
\dot{u}(x, y) \stackrel{\Delta}{=} a(x, y) e^{j \theta(x, y)}=\sum_{\left(x^{\prime}, y^{\prime}\right) \in R(x, y)} \frac{\left\|\boldsymbol{u}(x, y)-\boldsymbol{u}\left(x^{\prime}, y^{\prime}\right)\right\|}{B \cdot \operatorname{sign}\left(x, y, x^{\prime}, y^{\prime}\right)} \cdot\left[\left(x^{\prime}-x\right)+j\left(y^{\prime}-y\right)\right]
$$

where $B$ is the number of spectral bands (1 for monochrome images), $\boldsymbol{u}(x, y)$ represents pixel $(x, y)(\mathrm{a}$ scalar for monochrome images and a $B$-element vector for multi-band images), and $R(x, y)$ is the 8 neighborhood of pixel $(x, y)$. sign $\left(x, y, x^{\prime}, y^{\prime}\right)$ is the sign of the difference between the sum of the elements of the vector $\boldsymbol{u}(x, y)$ and the sum of the elements of vector $\boldsymbol{u}\left(x^{\prime}, y^{\prime}\right) . a(x, y) \geq 0$ and $\theta(x, y) \in[-\pi, \pi]$ are the Prewitt derivative amplitude and phase. In Prewitt edge detection, pixel $(x, y)$ is said to be an edge pixel if $a(x, y)>a_{0}$ (an amplitude threshold) and $a(x, y)$ is a local maximum along the direction of the phase $\theta(x, y)$

. For 8-neighborhoods, $(x, y)$ is thus an edge pixel if

$$
\begin{aligned}
& a(x, y)>\max \left[a_{0}, a\left(x+\Delta_{x^{\prime}} y+\Delta_{y}\right), a\left(x-\Delta_{x}, y-\Delta_{y}\right)\right] \\
& {\left[\Delta_{x}, \Delta_{y}\right]= \begin{cases}{[1,0]} & \theta^{\prime}(x, y)<\pi / 8 \text { or } \geq 7 \pi / 8 \\
{[1,1]} & \pi / 8 \leq \theta^{\prime}(x, y)<3 \pi / 8 \\
{[0,1]} & 3 \pi / 8 \leq \theta^{\prime}(x, y)<5 \pi / 8 \\
{[1,-1]} & 5 \pi / 8 \leq \theta^{\prime}(x, y)<7 \pi / 8\end{cases} } \\
& \theta^{\prime}(x, y) \stackrel{\Delta}{=}\left\{\begin{array}{ll}
\theta(x, y) & \theta(x, y) \geq 0 \\
\theta(x, y)+\pi & \theta(x, y)<0
\end{array} \in[0, \pi]\right.
\end{aligned}
$$

A very dense edge image can be obtained by choosing $a_{0}=0$ (as in our examples). We chose to completely thin the resulting edges morphologically.

The phase-based road detection algorithm decomposes a very dense edge image into separate channels, each containing edge pixels whose phases lie within a different range of orientations. There are $N$ channels corresponding to target phases of $\phi_{n}=n \Delta_{\phi}, n=0 \ldots N-1$, where $\Delta_{\phi}=\pi / N$ is the channel angular increment. To minimize $N$ (and thus computational complexity), $\Delta_{\phi}$ should be chosen as large as possible, but such that further reductions in $\Delta_{\phi}$ have negligible impact on road detection. For example, we have empirically established that $\Delta_{\phi} \approx 5^{\circ}(N \approx 36$ channels $)$ is sufficient for images that are roughly $1024 \times 1024$ in size. Edge pixel $(x, y)$ is assigned to channel $n$ if edge pixel phase is within a tolerance of channel $n$ target phase, i.e., 


$$
\left|\cos \left[\theta(x, y)-\phi_{n}\right]\right| \geq \cos \Delta_{\theta}
$$

for some angular consistency threshold $\Delta_{\theta} \in[0, \pi / 2]$. If too restrictive a threshold is imposed ( $\Delta_{\theta}$ is chosen too close to zero), the edge channels will tend to have too few edge pixels and the edge curves will tend to be broken because edge phases tend to be noisy. If too loose a threshold is imposed ( $\Delta_{\theta}$ is chosen too close to $\pi / 2$ ), the edge channels will tend to contain not only edge pixels with phases close to channel target phase, but also many edge pixels with phases far from channel target phase, which defeats the purpose of creating separate channels in the first place. Roadmaps tend to become busier as $\Delta_{\theta}$ increases. We have empirically established that $\Delta_{\theta}<10^{\circ}$ is typically too restrictive, and use $\Delta_{\theta}=20^{\circ}$ in all our examples.

Phase-based road detection is based on the idea that roads can typically be modeled as sets of extended line or curve segments that are connected. Since connected edges in channel $n$ correspond to line or curve segments at orientation $\phi_{n}$, one might expect to be able to detect roads by extracting extended line or curve segments from each edge channel and merging the results into a composite roadmap. Extended line or curve segments can be extracted from an edge channel by applying a decluttering process that removes edge segments with less than $N_{\text {channel }}$ pixels. Once the de-cluttered edge channels are recombined, the composite roadmap can be separately de-cluttered by removing edge segments with less than $N_{\text {composite }}$ pixels. The composite roadmap will be unaffected if $N_{\text {composite }} \leq$ $N_{\text {channel }} N_{\text {channel }}$ and $N_{\text {composite }}$ are user-supplied parameters for which smaller values tend to produce busier roadmaps. Fig.1 provides a graphical depiction of the phase-based road detection process.

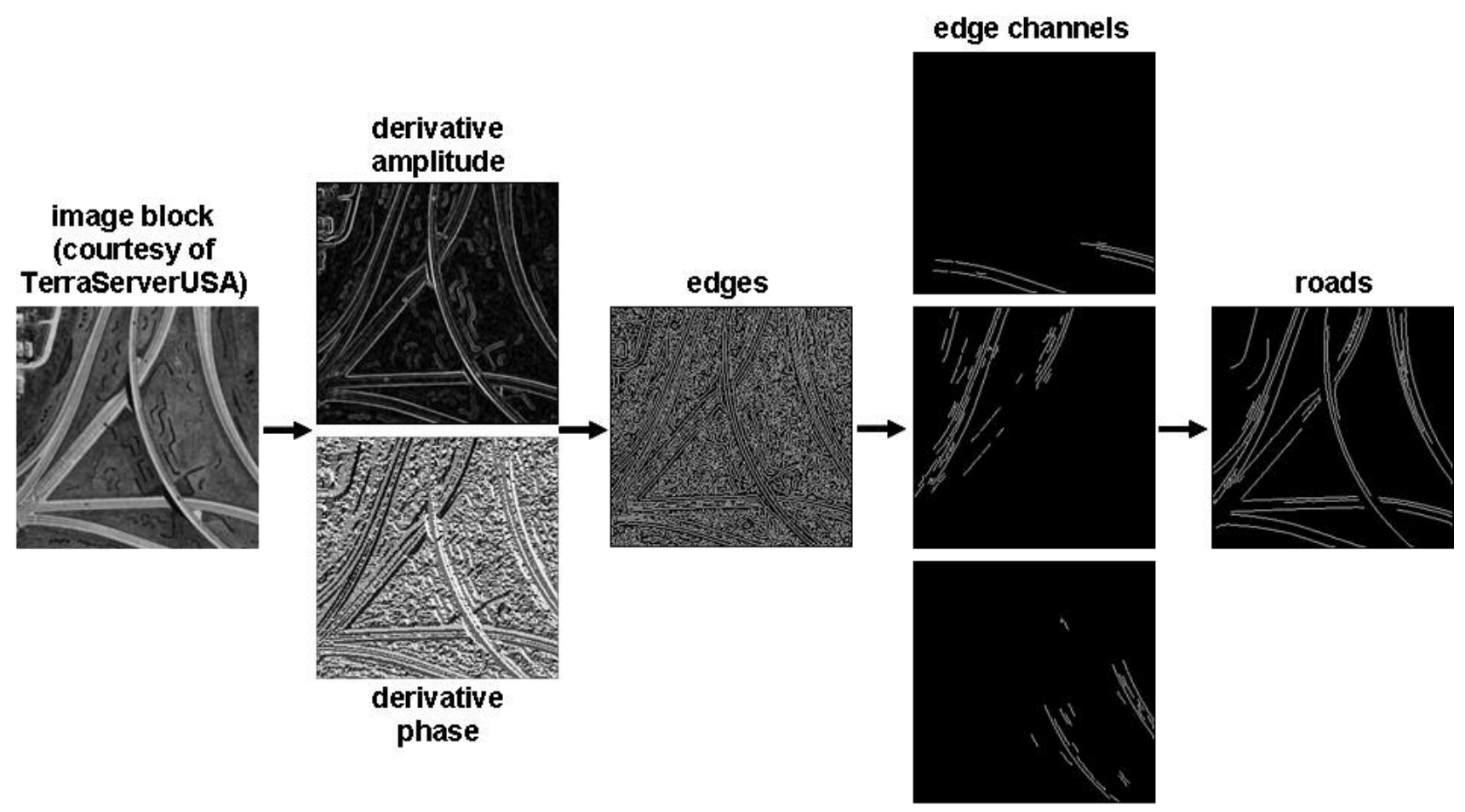

Fig.1 Graphical depiction of the phase-based road detection process. 


\section{EXAMPLES}

Fig.2 shows a TerraServerUSA image of Omaha (urban) and a DigitalGlobe image of a desert, along with associated roadmaps obtained by applying the phase-based road detection algorithm. In each case, $\left[N_{\text {channel }} N_{\text {composite }}\right]=[10,20]$.

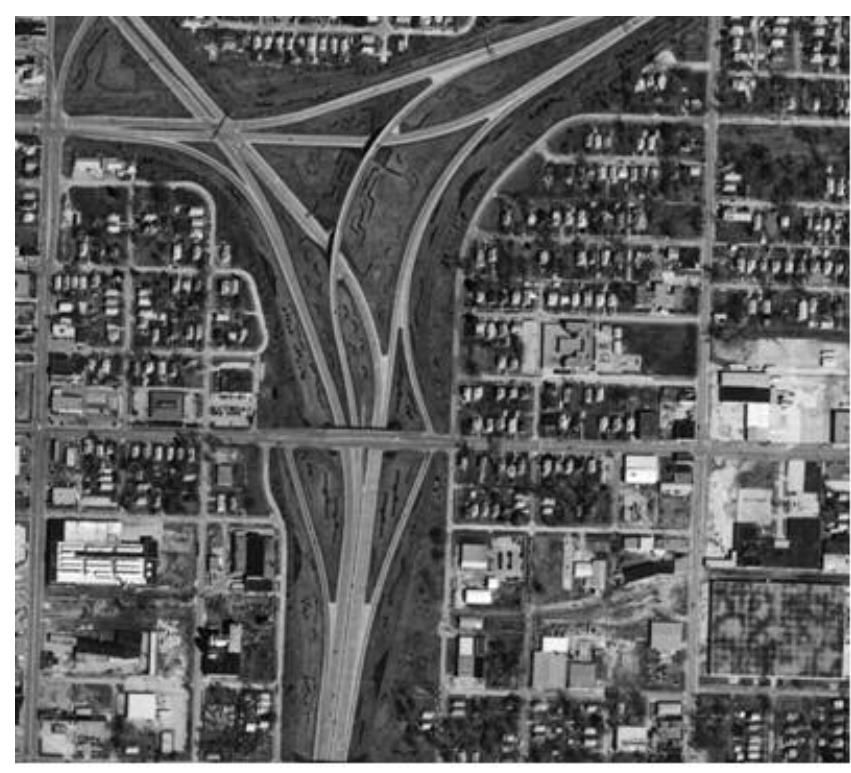

(a)

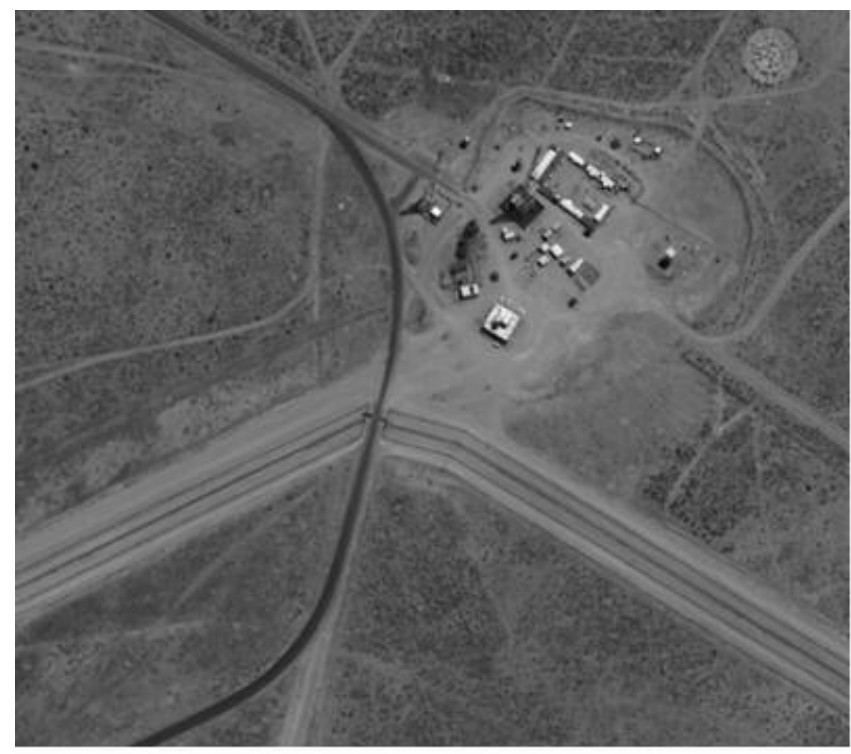

(c)

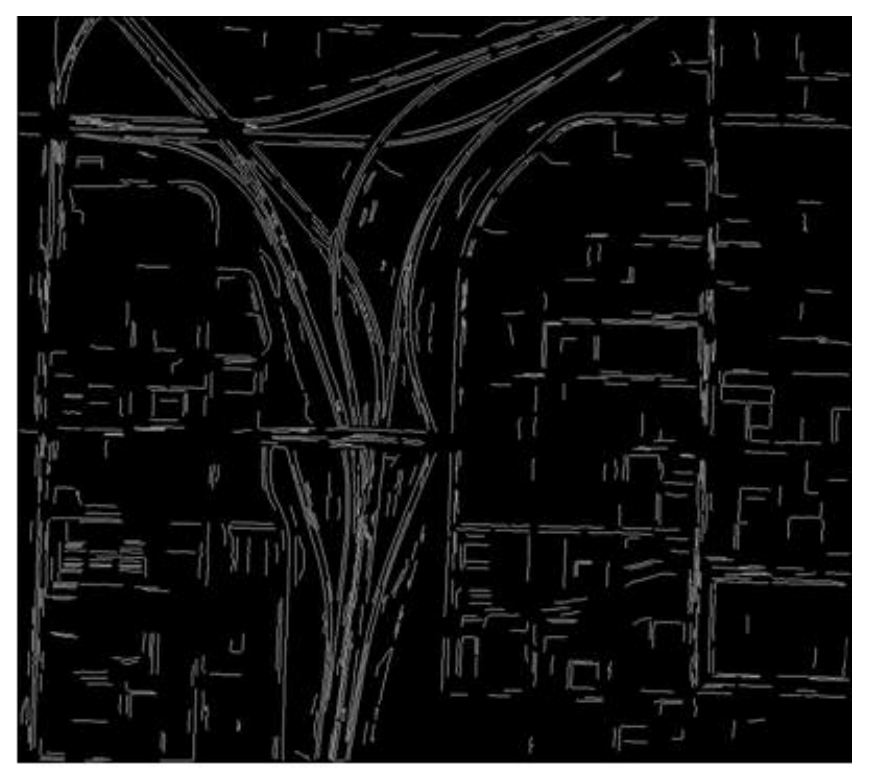

(b)

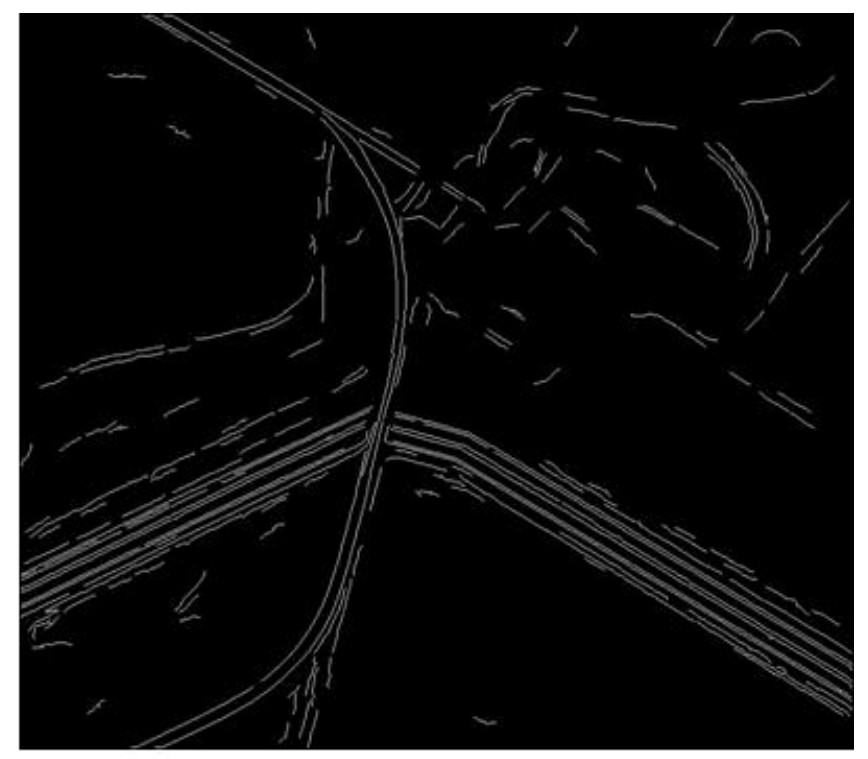

(d)

Fig.2 (a) Omaha image (courtesy of TerraServerUSA) (b) Omaha roadmap (c) Desert image (courtesy of DigitalGlobe) (d) Desert roadmap. 


\section{ROAD REPRESENTATIONS}

The phase-based road detector produces low level representations of roads, namely maps of road pixels. Although this might be sufficient if the objective is to render detected roads, higher level road representations are needed if the objective is to find the distance from a suspected object to the nearest road or to update a map of road networks stored in a GIS.

Distance maps (arrays of distances from each pixel to the nearest road pixel) explicitly support the objective of measuring distance to roads, and there are efficient algorithms for generating distance maps from binary images (e.g., [9]). Distance maps provide a slightly higher level description of road networks than road maps, but require more memory.

Road and distance maps constitute raster representations of roads. Vector representations are more compatible with GIS's and more compact. The simplest vector representations are lists of road pixels. The notion of road pixels can be generalized to rectangular road tiles with multiple pixels. Road tiles must contain sufficient numbers of edge pixels from a single orientation channel with relatively few edge pixels from other channels. One might also require road tiles to cover roads of prescribed widths. The attributes of a road tile might thus include position, dimensions, road orientation, road width and road centroid. Road tiles provide a mechanism for connecting roads that contain disconnected edge pixels. They also provide a mechanism for generating polyline representations for roads (as in GIS's). The polyline vertices are road centroid attributes of road tiles. Two road centroids are connected if the two associated road tiles touch and have compatible road orientations.

\section{SUMMARY AND FUTURE WORK}

A novel efficient method for automatically generating low level representations of roads directly from digital multi-source images based on edge phase has been introduced and demonstrated. Future work will address techniques for connecting disconnected curve segments so that road map clutter can be further reduced. Also, methods for migrating from raster (road image) to vector (polyline) representations of roads will be pursued.

\section{SELECTED REFERENCES}

[1] A. K. Shackelford and C. H. Davis, "A Combined Fuzzy Pixel-Based and Object-Based Approach for Classification of High-Resolution Multispectral Data over Urban Areas", IEEE Trans. Geosci. Remote Sens., Vol.41, No.10, October 2003, pp.2354-2363.

[2] D. Gabor, "Theory of Communication", J. IEE (London), vol.93, 1946, pp.429-457.

[3] D. Dunn, W. Higgins and J. Wakeley, "Texture Segmentation using 2D Gabor Elementary Functions", IEEE Trans. Pattern Anal. Mach. Intell., vol.16, February 1994, pp.130-149.

[4] B. S. Manjunath and W. Y. Ma, "Texture Features for Browsing and Retrieval of Image Data", IEEE Trans. PAMI, vol.18, no.8, August 1996, pp.837-842.

[5] P. V. C. Hough, "Method and Means of Recognizing Complex Patterns", U.S. Patent 3,069,654, 1962.

[6] D. Marr, E. Hildreth, "Theory of Edge Detection”, Proc. Roy. Soc. London, B207, 1980, pp.187217.

[7] J. Canny, "Computational Approach to Edge Detection", IEEE Trans. PAMI, Vol.8, No.6, November 1986, pp.679-698.

[8] J. M. S. Prewitt, "Object Enhancement and Extraction", in Picture Processing and Pyschopictorics, B. S. Lipkin and A. Rosenfeld, eds., Academic Press, New York, 1970.

[9] D. W. Paglieroni, "A Unified Distance Transform Algorithm and Architecture", Machine Vision and Applications, vol.5, 1992, pp.47-55. 